\title{
Evaluation of Equivalent Circuit Diagrams and Transfer Functions for Modeling of Lithium-Ion Batteries
}

\author{
Ahmad Rahmoun (Researcher, Institute for Applied Battery Research - IABF), Helmuth Biechl (Director, IABF) \\ and Argo Rosin (Senior Research Scientist, Tallinn University of Technology)
}

\begin{abstract}
The rapid developments in the field of electrochemistry, enabled lithium-ion batteries to achieve a very good position among all the other types of energy storage devices. Therefore they became an essential component in most of the modern portable and stationary energy storage applications, where the specific energy and the life time play an important role. In order to analyze and optimize lithium-ion batteries an accurate battery model for the dynamic behavior is required. At the beginning of this paper four different categories of electrical models for li-ion cells are presented. In the next step a comparison between equivalent circuit diagrams and fractional rational functions with the complex variable $s$ is shown for lithium-ion battery modeling. It is described how to identify the parameters of the models in the time domain and also in frequency domain. The validation of the different models is made for high and low dynamic current profiles. In the first step the dependency of all model parameters on the temperature and on the battery age is neglected. These effects will be taken into account in the continuation of this work.
\end{abstract}

Keywords - Batteries, equivalent circuits, mathematical model, transfer functions.

\section{INTRODUCTION}

In order to analyze and optimize systems with Li-ion batteries, such as smart grids with big energy storage devices, photovoltaic, and other systems, it is necessary to have accurate electrical models of all the components in the system. Regarding Li-ion cells the dynamic behavior depends on SOC, internal temperature and aging. Therefore all three effects should be taken into account. An accurate model must produce (over the whole life time) a precise prediction of the output voltage during different discharge/charge current rates, and ambient temperatures of the battery. Additional models are needed for the accurate estimation of other battery parameters such as state of charge (SOC), internal temperature and state of health (SOH).

By having battery models that can fulfill all the previous requirements, storage system designers can predict and optimize system performance under different conditions just by simulation, and therefore they can reduce the experimental efforts and time [1], [2].

Modeling of $\mathrm{Li}$-ion batteries is not an easy task due to the following reasons $[3]-[6]$ :

- Li-ion batteries are complex systems due to SOC variation, diffusion phenomenon and different aging effects.

- They are highly nonlinear systems regarding SOC, temperature, and aging.
In the way to develop an accurate model for Li-ion batteries both time and frequency domain behaviors of the model should be investigated thoroughly. For this purpose two groups of models have been investigated in this paper. The first group contains the equivalent circuit models with RLC elements which can be easily identified from the time domain measurements. The second group consists of fractional rational functions representing a good method to fit the battery impedance spectrum curves in frequency domain.

This paper is structured as follows: Section II presents a brief state of art analysis of the different model architectures used to model Li-ion batteries; Section III explains the two types of the identification methods and how to be applied to the selected models. Section IV shows the experimental setup and experimental results used to validate the models, Section $\mathrm{V}$ concludes this paper.

The research work which is published in the paper is a step forward on the way to simulate a big energy storage system in the research project "IRENE" (Integration of Renewable Energies and Electromobility). This energy storage system (300kW, 170kWh) includes also inverters, transformers, filters, and other power electronic devices [7].

\section{BATTERY MODELS}

The first step in the modeling process is always to choose the right model structure which is able to represent the physical phenomena which occur inside the battery. However the main difficulty in this process is always how to compromise between model accuracy and complexity. In other words the model must be accurate enough to fit the dynamic requirements of the application, and at the same time it should be as simple as possible.

This compromise between accuracy and complexity has pushed the researchers to find out many model different structures. These structures can be categorized according to the following four aspects:

\section{A. Full Parametric Model}

It describes mathematically the whole behavior of the cell including the open circuit voltage (OCV) as a function of SOC, hysteresis and cell temperature. This kind of model is very suitable for parameter and state estimation purposes as shown in [8] - [10], where five mathematical state-space structures have been introduced as follows:

- Combined model 
- Simple model

- Zero-State hysteresis model

- One-State hysteresis model

- Enhanced self-correcting (ESC) model

The parameters of these models are identified by the use of Extended Kalman Filter (EKF), and then a comparison between the simulation and measurement results is made by calculating the average of the root mean value of the error (RMSE). Ref. [9] depicts that the simple model has the highest RMSE of $53.8 \mathrm{mV}$ over temperature range between 30 and $45^{\circ} \mathrm{C}$, the best model according to this study is the ESC model which can achieve a RMSE of $11,8 \mathrm{mV}$ over the same temperature range. Similar results can be also extracted from [10]. The main advantage of this type of models is the small number of parameters to model the cell.

\section{B. Equivalent Circuit Model with RLC Elements}

It uses a network of $\mathrm{R}, \mathrm{L}$ and $\mathrm{C}$ elements to simulate the dynamic characteristics of the Li-ion cell, and a voltage source to model the OCV as shown in Fig.1, demonstrating one time constant (OTC) model and two time constants (TTC) model [11]. This type of models can afford a better accuracy compared to the full parametric models [12]. On the other hand they need larger memory size to store the model parameters and the OCV look up table. So they are compromising between the model accuracy and memory size.

Ref. [12] introduces a comparative study between twelve model structures for li-ion batteries, the twelve model structures include the five structures which have been already introduced in the last paragraph, beside the equivalent circuit model structures with one, two, three time constants, and taking into account with or without hysteresis effect. The model parameters are identified in time domain using multiswarm particle swarm optimization (MPSO) method.

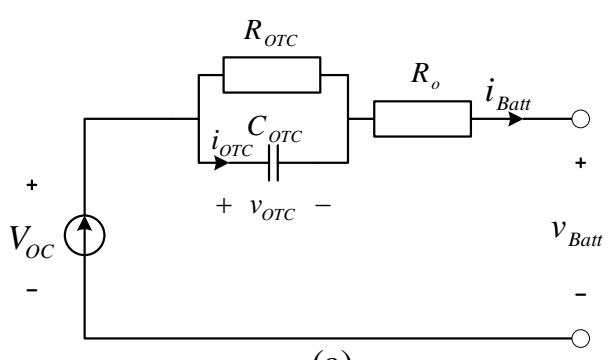

(a)

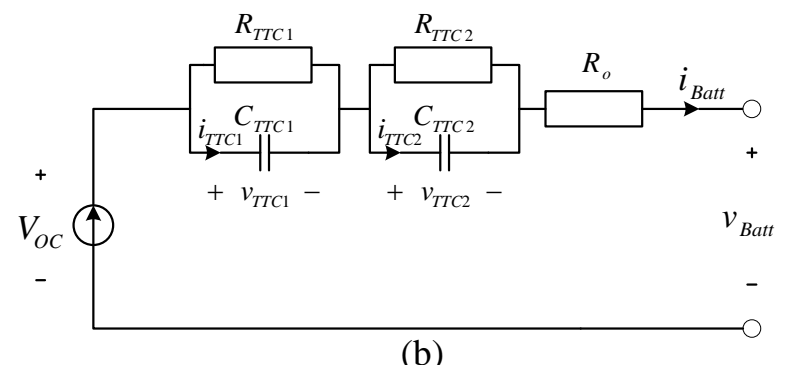

(b)

Fig.1. Battery equivalent circuit diagrams: (a) one time constant model (OTC), (b) two time constants model (TTC).
Ref. [12] indicates that the equivalent circuit models with RC elements can afford a lower RMSE than the full parametric models for $\mathrm{LiNMC}$ and $\mathrm{LiFePO}_{4}$ cells.

\section{Equivalent Circuit Models with Zarc and Warburg Elements}

The idea behind this type of models is to simulate the behavior of the Li-ion cell in both time and frequency domains very precisely. To achieve this goal new elements have been introduced in the frequency domain such as the constant phase element (CPE), the Zarc element, and the Warburg element. These elements do not have a Laplace transformation, thus it is not possible to have a representation of them in time domain without approximations [13].

Ref. [13] shows also a comparison between an equivalent circuit model with RLC elements and an equivalent circuit model using Zarc and Warburg elements by applying different test profiles to the cell at different temperatures. Subsequently the measured output voltage is compared to the calculated output of both models. As a result of the comparison both models can produce a very satisfying agreement with the measured values. In opposite the equivalent circuit model with Zarc and Warburg elements is able to reproduce the impedance spectra more accurately than the equivalent circuit model with RLC elements at the cost of double of the computation time.

\section{Fractional Rational Functions}

This is our new approach to find out an optimum between model accuracy and complexity. This approach imposes that the battery model should match the time and frequency domain behavior of Li-ion battery as accurate as possible without using any complex structures.

Fractional rational functions can be used as a general concept for modeling any linear system in the frequency domain. They have been used extensively in many applications [14]. Therefore one can get benefit from the high number of literature sources and applications in this field. The fractional rational function is defined as shown in (1), $n_{b}$ and $\mathrm{n}_{\mathrm{a}}$ are numerator and denominator orders respectively (where $\mathrm{a}_{0} \neq 0$ and $\left.\mathrm{b}_{0} \neq 0\right), \quad\left\{\mathrm{b}_{0}, \mathrm{~b}_{1}, \cdots, \mathrm{b}_{\mathrm{nb}}\right\}$ and $\left\{\mathrm{a}_{0}, \mathrm{a}_{1}, \ldots, \mathrm{a}_{\mathrm{na}}\right\}$ are the numerator and denominator coefficients respectively.

$$
H(s)=\frac{B(s)}{A(s)}=\frac{b_{0} s^{n_{b}}+b_{1} s^{n_{b}-1}+\cdots+b_{n_{b}}}{a_{0} s^{n_{a}}+a_{1} s^{n_{a}-1}+\cdots+a_{n_{a}}} .
$$

The advantage of using fractional rational functions for modeling of Li-ion cells mainly is flexibility. Fractional rational functions do not need complex optimization methods to estimate model parameters. The model parameters can be identified in the frequency domain but also in the time domain where the state space representation is used and the same coefficients occur [15]. Above all the model accuracy can be improved by increasing the order of polynomials in the numerator and denominator. Hence the fractional rational functions are a good choice to model the frequency response of a battery and thus the dynamic behavior in the range where it can be treated as being linear. 


\section{IDENTIFICATION METHODS}

Identification methods can be divided mainly into two branches as follows:

\section{A. Time Domain Identification Methods}

These methods consist of applying a current signal with a defined profile to the battery, and measuring the output voltage. The current profile can be a pulse with a high current value or it can be a pseudo random binary sequence. These methods have the advantage of the possibility to use high current values during modeling; on the other hand the frequency range might be limited by the sampling frequency and therefore the model at high frequencies cannot be identified accurately enough.

\section{B. Frequency Domain Identification Methods}

These methods employ the electrochemical impedance spectroscopy (EIS) technique to extract the complex impedance values over a certain frequency range. Subsequently the impedance curves are fitted to a variety of model structures by using the nonlinear complex least square (NCLS) optimization method. The EIS technique affords a precise impedance measurement in a wide band of frequencies but only for low current values [13], [16].

The next paragraphs show a description of two different model structures, and the identification methods which have been used to adapt the model parameters.

\section{Parameters Identification of the RC Elements of an Equivalent Circuit Model}

The identification of the model parameters is made by applying a current signal to the battery with rectangular shape with short and long interruptions. At the same time the battery output voltage is measured. The ohmic resistance $R_{0}$ of the battery is calculated during short interruptions of the current signal, while OTC and TTC model parameters are estimated during long interruptions using nonlinear least squares algorithm [11].

\section{Parameters Identification of Fractional Rational Function}

By applying the EIS technique to the battery, complex values of the impedance are obtained for discrete frequencies. These values form the battery frequency response. The adaptation of the model parameters is made by using the Matlab function "invfreqs". This function finds the continuous-time transfer function $\mathrm{H}(\mathrm{s})$ corresponding to a given complex frequency response with $\mathrm{n}$ frequency pins. "invfreqs" uses the equation error in (2) to optimize the fitting in order to find the best model which fits the data.

$$
\min _{a, b} \sum_{k=1}^{n} w_{k}\left|h_{k} A\left(\omega_{k}\right)-B\left(\omega_{k}\right)\right|^{2},
$$

where: $\left\{\mathrm{w}_{\mathrm{k}}: \mathrm{k}=1,2, \ldots, \mathrm{n}\right\}$ are the weighting factors. After obtaining the transfer function coefficients, $\left\{\mathrm{b}_{0}, \mathrm{~b}_{1}, \ldots, \mathrm{b}_{\mathrm{nb}}\right\}$ and $\left\{\mathrm{a}_{0}, \mathrm{a}_{1}, \ldots, \mathrm{a}_{\mathrm{na}}\right\}$ the continuous time transfer function $\mathrm{H}(\mathrm{s})$ is converted into discrete time transfer function $\mathrm{H}(\mathrm{z})$ by using of the Matlab function "c2d" with the sampling time Ts=100ms.
Finally the discrete time transfer function $\mathrm{H}(\mathrm{z})$ is simulated by using Matlab function "lsim”.

This identification process has been applied to the battery impedance measurements in order to calculate the parameters of a third order transfer function $\left(\mathrm{TF}_{3}: \mathrm{n}_{\mathrm{b}}=\mathrm{n}_{\mathrm{a}}=3\right)$ and a sixth order transfer function $\left(\mathrm{TF}_{6}: \mathrm{n}_{\mathrm{b}}=\mathrm{n}_{\mathrm{a}}=6\right)$.

\section{VALIDATION OF THE MODELS}

For the validation experiment a lithium polymer battery cell has been used, the important cell data are depicted in Table I.

The validation criterion consists of two stages. In first stage the EIS meter measurements respectively the current pulse is applied to the battery in order to identify the parameters of models concerning equivalent circuit diagrams respectively fractional rational functions. Fig.2 and Fig.3 show the measured Nyquist and bode plots of the battery impedance and the frequency responses of the models. The sixth order transfer function shows the best fit to the impedance curve. The third order transfer function comes in the second place; it shows a rough approximation of the battery. Both equivalent circuit diagrams OTC and TTC show an analogue behavior of the battery but only for low frequencies.

The second stage includes applying a current profile with two phases (phase-I and phase-II) to the battery. During this time the output voltage is acquired with the same sampling time Ts. The first phase is dedicated to test the transient response of the different models for the high dynamic current profiles, and the second phase is dedicated to test the steady state error of the models.

Phase-I of the current profile is a random pseudo sequence of current pulses with a mean value of zero. The maximum absolute value of the current pulses is 40A. Each current pulse has the duration of $1 \mathrm{~s}$, and the number of pulses is 120 , which results in 120 seconds for the whole duration of phase-I.

Phase-II consists of a current pulse with constant value of 20A for duration of 10 minutes. This duration is chosen to be long enough to test the models error in the steady state and short enough to hold the deviation of SOC value within appropriate limits (otherwise the models require a SOC adaptation). Phase-I and Phase-II represent the high and low dynamical load with respect to the electrical behavior.

TABLE I

LFP CELL DATA

\begin{tabular}{|l|l|l|}
\hline \multicolumn{2}{|l|}{ Typical Capacity } & $40 \mathrm{Ah}$ \\
\hline \multicolumn{2}{|l|}{ Rated Voltage } & $3.2 \mathrm{~V}$ \\
\hline \multirow{2}{*}{$\begin{array}{l}\text { Charge } \\
\text { Condition }\end{array}$} & Max. Current & $120 \mathrm{~A}$ \\
\cline { 2 - 3 } & Voltage & $4.0 \mathrm{~V}$ \\
\hline \multirow{3}{*}{$\begin{array}{l}\text { Discharge } \\
\text { Condition }\end{array}$} & Continuous Current & $120 \mathrm{~A}$ \\
\cline { 2 - 3 } & Peak Current & $800 \mathrm{~A}$ \\
\cline { 2 - 3 } & Cut-off Voltage & $2.8 \mathrm{~V}$ \\
\hline
\end{tabular}




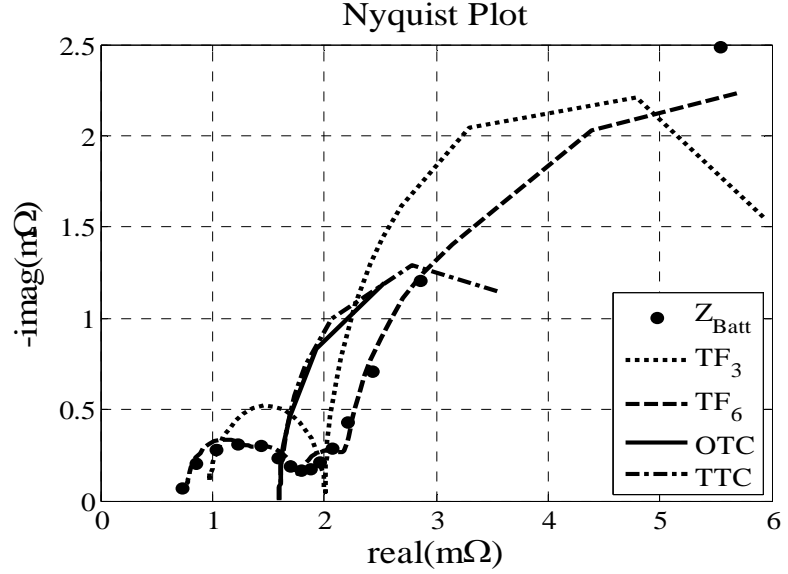

Fig.2. Nyquist plot of the battery electrochemical impedance measurements and the frequency response of the $\mathrm{TF}_{6}, \mathrm{TF}_{3}$, OTC and TTC models.
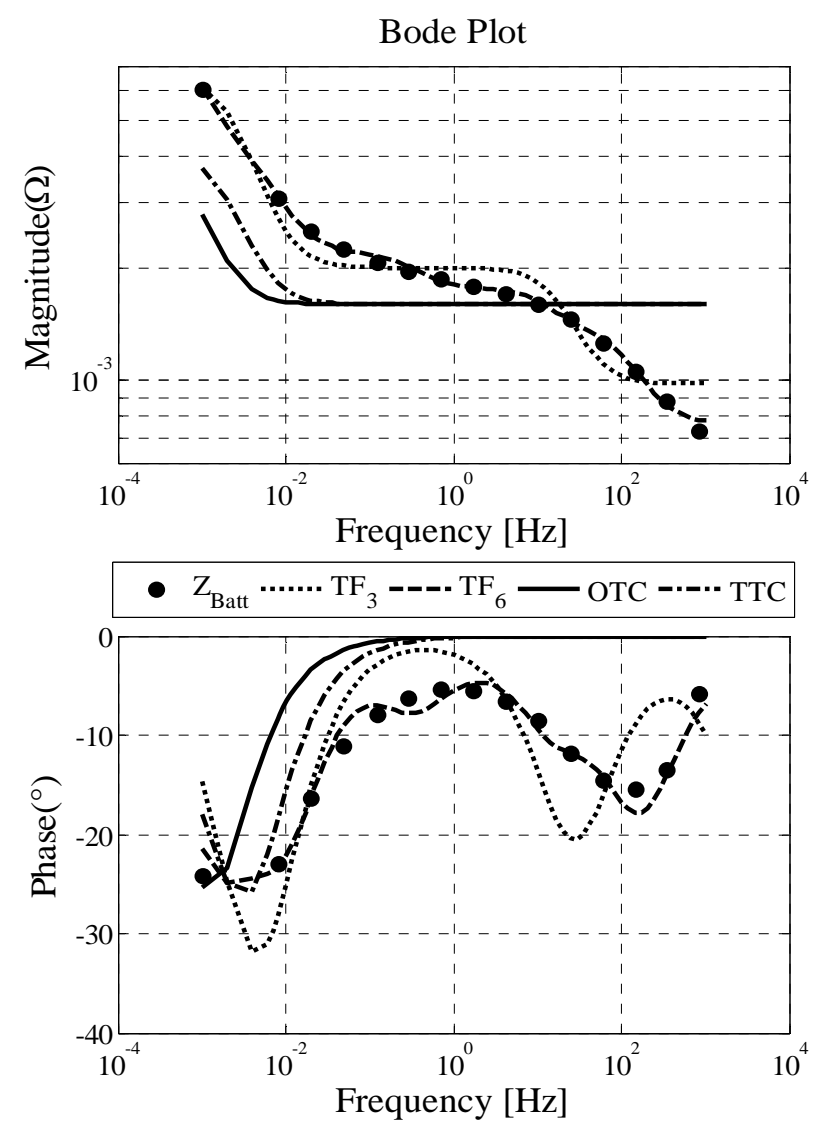

Fig.3. Bode plot of the battery electrochemical impedance and the measurements frequency response of the $\mathrm{TF}_{6}, \mathrm{TF}_{3}$, OTC and TTC models.
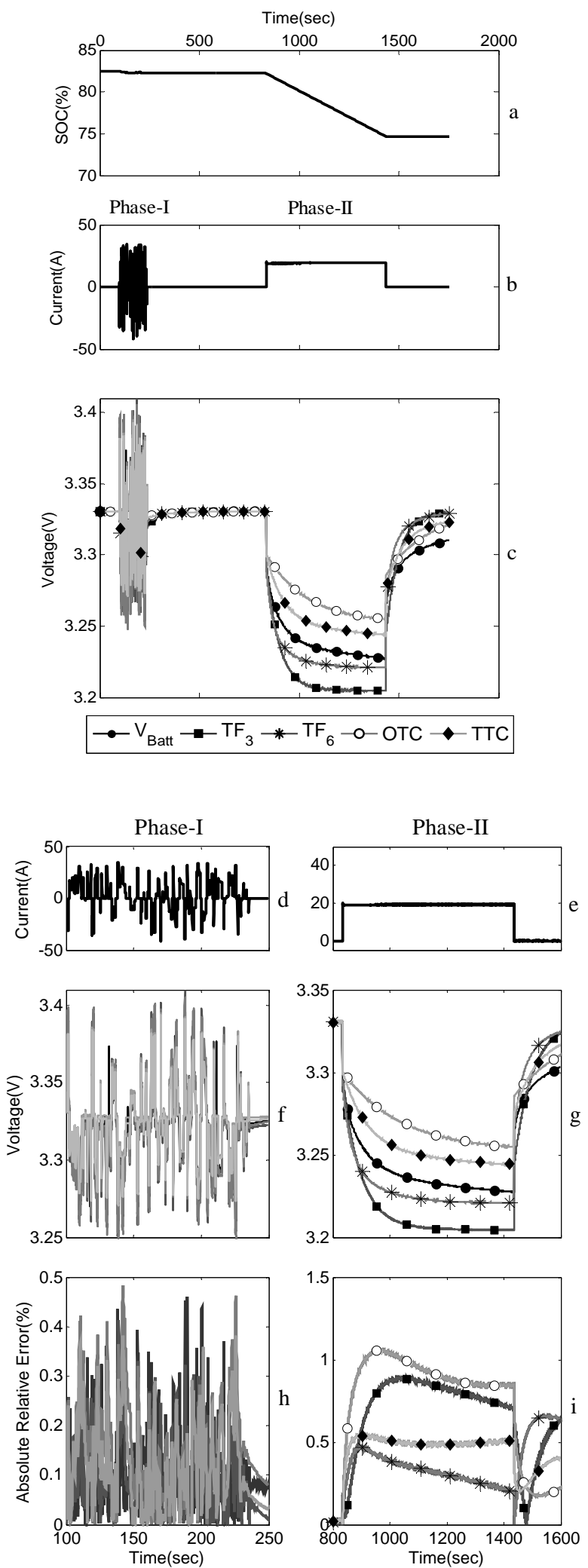

Fig.4. Experimental results for battery model validation: (a) battery state of charge during the test, (b) impressed current profile which is applied to the battery, (c) measured battery output voltage, (d) and (e) - detailed battery current profile during phase-I and phase-II respectively, (f) and (g) - measured battery voltage and calculated model outputs in detail during phase-I and phase-II respectively, (h) and (i) - relative error in percentage calculated for all models during phase-I and phase-II respectively. 


\section{Maximum Absolute Value of Relative Error (\%)}

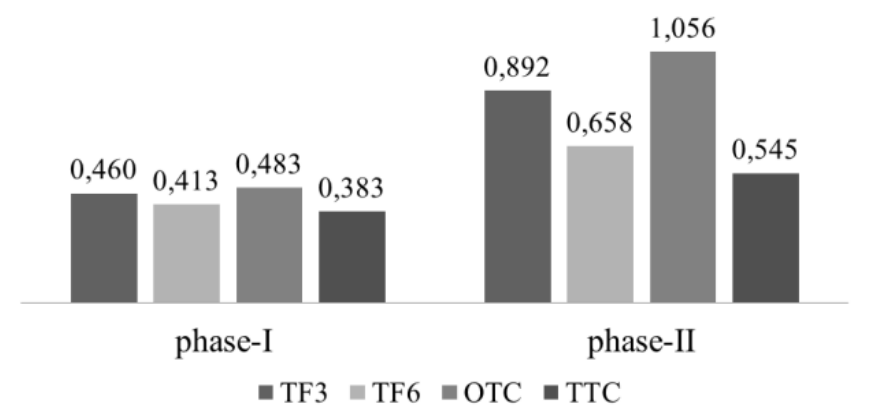

Fig.5. Comparison between the maximum absolute values of the relative error of the $\mathrm{TF}_{6}, \mathrm{TF}_{3}$, OTC and TTC models during phase-I and phase-II.

Average of Absolute Value of Relative Error

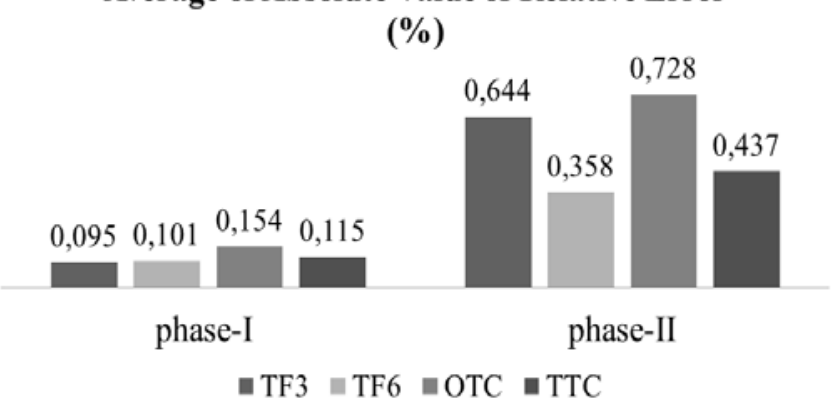

Fig.6. Comparison between the average absolute values of the relative error of the $\mathrm{TF}_{6}, \mathrm{TF}_{3}$, OTC and TTC models during phase-I and phase-II.

Phase-I and phase-II are repeated sequentially for several SOC values. Fig.4 demonstrates the experimental and calculation results around an SOC value of $80 \%$. Fig.4(a) shows that the SOC value calculated during phase-I and phase-II is changing in the range of less than $5 \%$. Thus it can be assumed to be more or less invariant. The current profile is shown in Fig.4(b), while Fig.4(d) and (e) show the impressed battery output current during phase-I and phase-II respectively. In Fig.4(c) one can see the measured output voltage of the battery and the calculated output voltage resulting from the different models. In Fig.4(f) and (g) the transient behavior during phase-I and phase-II respectively can be seen in detail.

The absolute value of the relative error in Fig.4(h) and 4(i) during phase-I and phase-II respectively shows that the relative error during phase-I is less than $0.5 \%$, and less than $1.5 \%$ during phase-II.

\section{V.CONCLUSION}

This paper presents firstly a brief explanation about different types of model structures for Li-ion cells. Secondly a comparison between two different model structures has been carried out. The sixth order and third order transfer functions show a very good fit to the electrochemical impedance measurements of the battery which has resulted in very small relative error values during phase-I as shown in Fig.5 and Fig.6. During phase-II thermal effects grow up and affect the dynamical characteristics of the battery, hence the error increases for both $\mathrm{TF}_{6}$ and $\mathrm{TF}_{3}$. Equivalent circuit diagrams show a worse fit to the battery electrochemical impedance measurements of the battery, but they have a stable performance over phase-I and phase-II. This stability in performance is due to the fact that the identification process of the equivalent circuit diagrams is made under similar conditions concerning internal temperature and current value.

In a continuation of this research the effect of current value, internal temperature and aging will be taken into account.

\section{ACKNOWLEDGMENT}

The authors wish to thank the Federal Ministry of Economics of Germany for the financial support by the research project IRENE (Integration of Renewable Energy and Electric Mobility). Further many thanks to Prof. Dr.-Ing. A. Rupp, Vice President for R\&D of the University of Applied Sciences Kempten, for his continuous support of the Institute for Applied Battery Research.

This Estonian partner research work has been supported by Estonian Ministry of Education and Research (Project SF0140016s11), and Estonian Science Foundation (Grant No. 9350).

\section{REFERENCES}

[1] Huria, T.; Ceraolo, M.; Gazzarri, J.; Jackey, R., "High fidelity electrical model with thermal dependence for characterization and simulation of high power lithium battery cells," Electric Vehicle Conference (IEVC), 2012 IEEE International , vol., no., pp.1,8, 4-8 March 2012

[2] Min Chen; Rincon-Mora, G.A., "Accurate electrical battery model capable of predicting runtime and I-V performance," Energy Conversion, IEEE Transactions on , vol.21, no.2, pp.504,511, June 2006

[3] M. Montaru and S. Pelissier, "Frequency and Temporal Identification of a Li-ion Polymer Battery Model Using Fractional Impedance," in Les Rencontres Scientifiques de l'IFP - Advances in Hybrid Powertrains, Rueil-Malmaison, France, November 2008.

[4] S. Buller, M. Thele, E. Karden and R. W. De Doncker, "Impedancebased non-linear dynamic battery modeling for automotive applications," Journal of Power Sources, vol. 113, no. 2, pp. 422-430, 27 January 2003.

[5] Buller, S.; Thele, M.; De Doncker, R.W.A.A.; Karden, E., "Impedancebased simulation models of supercapacitors and Li-ion batteries for power electronic applications," Industry Applications, IEEE Transactions on , vol.41, no.3, pp.742,747, May-June 2005

[6] Al Jed, H.; Mieze, A.; Vinassa, J. -M; Simon, R., "Mathematical modeling of aging factors for Li-ion battery cells," Vehicle Power and Propulsion Conference (VPPC), 2010 IEEE , vol., no., pp.1,4, 1-3 Sept. 2010

[7] "IRENE," [Online]. Available: http://www.projekt-irene.de/

[8] G. L. Plett, "Extended Kalman filtering for battery management systems of LiPB-based HEV battery packs Part 1. Background," Journal of Power Sources, p. 252-261, 2004.

[9] G. L. Plett, "Extended Kalman filtering for battery management systems of LiPB-based HEV battery packs Part 2. Modeling and identification," Journal of Power Sources, p. 262-276, 2004.

[10] Farag, M. S.; Ahmed, R.; Gadsden, S.A.; Habibi, S.R.; Tjong, J., "A comparative study of Li-ion battery models and nonlinear dual estimation strategies," Transportation Electrification Conference and Expo (ITEC), 2012 IEEE , vol., no., pp.1,8, 18-20 June 2012

[11] A. Rahmoun and H. Biechl, "Parameters Identification of Equivalent Circuit Diagrams for Li-Ion Batteries," in 2012 11th International Symposium PÄRNU, PÄRNU, Estonia, 2012.

[12] X. Hua, S. Li and H. Peng, "A comparative study of equivalent circuit models for Li-ion batteries," Journal of Power Sources, p. 359-367, 2012. 
[13] D. Andre, M. Meiler, K. Steiner, H. Walz, T. Soczka-Guth and D. U. Sauer, "Characterization of High-Power Lithium-Ion Batteries by Electrochemical Impedance Spectroscopy. II: Modelling," Journal of Power Sources, p. 5349-5356, 15 June 2011.

[14] Biechl, H.; Lorenzen, H. W.; Lubasch, R.; Prenner, H., "Transient Behaviour of a Current-Source Inverter-Fed Synchronous Motor with a Solid Rotor," Power Engineering Review, IEEE , vol.9, no.9, pp.52,52, Sept. 1989

[15] H. Biechl and H. Bischof, "Meßtechnische Ermittlung von Systemübertragungsfunktionen aus transienten Übertragungsverläufen von Systemanregung und - antwort - Anwendung auf Synchronmaschinen," etzArch.10, pp. 47-51, 1988 (in German).

[16] O. Obeidi and H. G. Schweiger, "Impedance Spectra of Lithium Ion Cells Influence of Temperature, State of Charge and Ageing," in Kronacher Impedanztage 2011, Kronacher, Germany, 2011.

A. Rahmoun M.Eng. received Dipl.Eng. in Electronic Systems from Higher Institute for Applied Sciences and Technology (HIAST), Damascus, Syria, in 2006, and finished his Master study of Electrical Engineering at the University of Applied Sciences Kempten, Germany, in 2012.

His employment experience included Digital Image and Signal Processing Lab (HIAST), and he is currently a research engineer at IABF at the University of Applied Sciences Kempten (Kempten, Germany), and a PhD student at Tallinn University of Technology (Estonia).

Postal address: University of Applied Sciences Kempten, Institute for Applied Battery Research (IABF), Bahnhofstraße 61, 87435 Kempten (Allgäu), Germany.

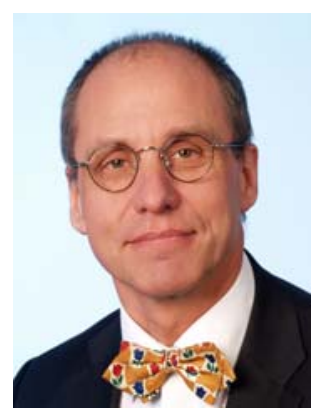

Helmuth Biechl received his Dipl.-Ing. Univ. and M.Sc. degree from the Technical University of Munich (TUM) in 1985 and his Dr.-Ing. and PhD degree in 1989 also from the Technical University of Munich. From 1989 till 1994 he was a developing and planning engineer and a group leader at the companies Siemens and E.ON.

Since 1994 he is a full professor at the University of Applied Sciences Kempten in Germany and working on the field of electrical power systems, mainly electrical machines, drives, power transportation and distribution systems and big batteries. Since 2011 he is the Director of the Institute for Electrical Power Systems. Since 2005 is a Titular Professor at the Universidad Pontificia Bolivariana in Colombia where he got a doctor honoris causa degree in 2012. His main research interests are modelling and simulation of electrical power systems. He has published more than 30 international scientific papers and about 200 articles in technical magazines.

Postal address: University of Applied Sciences Kempten, Institute for Applied Battery Research (IABF), Bahnhofstraße 61, 87435 Kempten (Allgäu), Germany.

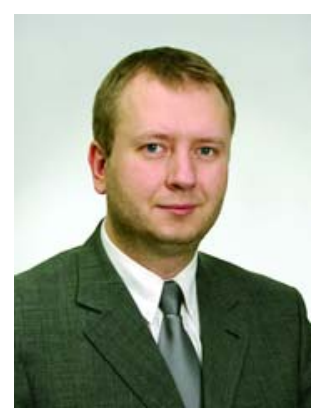

Argo Rosin received the Dipl. Eng., M.Sc. and Dr.Sc.techn. degrees in electrical engineering from Tallinn University of Technology, Tallinn, Estonia, in 1996, 1998 and 2005, respectively. He is presently a Senior Researcher in the Department of Electrical Engineering, Tallinn University of Technology. He has published more than 60 papers on energy management, control and diagnostic systems development and is the holder of a Patent in this application field. His research interests include modelling and simulation of power management and industrial control systems. He is member of Estonian Association of Engineers, Estonian Association of Transport and Roads.

Postal address: Faculty of Power Engineering, Tallinn University of Technology, Ehitajate str. 5, 19086 Tallinn, Estonia. 